\title{
On- and off-frequency compression estimated using a new version of the additivity of forward masking technique
}

\author{
Christopher J. Plack ${ }^{a)}$ \\ Human Communication and Deafness Division, University of Manchester, Manchester M13 9PL, United \\ Kingdom \\ Dhany Arifianto \\ Department of Engineering Physics, Sepuluh Nopember Institute of Technology, Surabaya 60111, Indonesia
}

(Received 3 November 2009; revised 24 May 2010; accepted 28 May 2010)

\begin{abstract}
On- and off-frequency compression at the 4000- and 8000-Hz cochlear places were estimated using a new version of the additivity of forward masking (AFM) technique, that measures the effects of combining two non-overlapping forward maskers. Instead of measuring signal thresholds to estimate compression of the signal as in the original AFM technique, the decrease in masker threshold in the combined-masker condition compared to the individual-masker conditions is used to estimate compression of the masker at the signal place. By varying masker frequency it is possible to estimate off-frequency compression. The maskers were 500-Hz-wide bands of noise, and the signal was a brief pure tone. Compression at different levels was estimated using different overall signal levels, or different masker-signal intervals. It was shown that the new AFM technique and the original AFM technique produce consistent results. Considerable compression was observed for maskers well below the signal frequency, suggesting that the assumption of off-frequency linearity used in other techniques may not be valid. Reducing the duration of the first masker from 200 to $20 \mathrm{~ms}$ reduced the compression exponent in some cases, suggesting a possible influence of olivocochlear efferent activity. (C) 2010 Acoustical Society of America. [DOI: 10.1121/1.3455844]
\end{abstract}

PACS number(s): 43.64.Kc, 43.66.Dc [MW]

Pages: 771-786

\section{INTRODUCTION}

Direct measurements of basilar membrane (BM) displacement or velocity in non-human mammals show that, in the basal (high-frequency) region of the cochlea, the BM response growth is strongly compressive at mid levels in response to tones close to the characteristic frequency $(\mathrm{CF})$ of each place (Rhode, 1971; Robles et al., 1986; Murugasu and Russell, 1995; Ruggero et al., 1997; Russell and Nilsen, 1997; Rhode and Recio, 2000). However, the response to tones well below $\mathrm{CF}$ is linear in the basal region (Ruggero et al., 1997; Russell and Nilsen, 1997). This finding has led to the development of behavioral techniques ${ }^{1}$ for measuring the BM response in humans. In these techniques, the effect of a forward masker below the signal frequency ("offfrequency" masker) is used as a "linear yardstick" for estimating the rate of growth of the on-frequency response. In the growth of masking (GOM) technique, masker level at threshold is measured as a function of signal level or vice versa (Oxenham and Plack, 1997; Hicks and Bacon, 1999; Moore et al., 1999; Rosengard et al., 2005). A plot of offfrequency masker level against signal level is an estimate of the on-frequency BM response function. In the temporal masking curve (TMC) technique, the masker level needed to just mask a low-level signal is measured as a function of the temporal gap between the masker and the signal (Nelson et al., 2001; Lopez-Poveda et al., 2003; Plack and Drga,

\footnotetext{
${ }^{\text {a) }}$ Author to whom correspondence should be addressed. Electronic mail chris.plack@manchester.ac.uk
}

2003; Rosengard et al., 2005; Williams and Bacon, 2005). A plot of off-frequency masker level against on-frequency masker level, paired by masker-signal interval, is an estimate of the on-frequency BM response function.

The assumption of linear BM processing for an offfrequency masker is problematic at low signal frequencies, which are represented near the apex of the cochlea. This is because compression at an apical location is applied to a wide range of frequencies above and below CF (Rhode and Cooper, 1996; Plack and Drga, 2003). It follows that the off-frequency masker cannot be used as a linear reference at low CFs. To avoid this problem, some researchers have used the off-frequency TMC (the plot of masker level at threshold against masker-signal interval) for a high signal frequency (e.g., $4000 \mathrm{~Hz}$ ) as a linear reference for estimating compression from the TMC for a low on-frequency masker (LopezPoveda et al., 2003; Plack and Drga, 2003; Nelson and Schroder, 2004; Lopez-Poveda et al., 2005; Williams and Bacon, 2005).

However, there is concern that, even at high CFs, the assumption of off-frequency linearity may not be valid for some of the masker frequencies used in previous studies. Plack et al. (2004) and Lopez-Poveda et al. (2005) reported that off-frequency TMCs for a $4000-\mathrm{Hz}$ signal were steeper for normal-hearing listeners than for listeners with sensorineural hearing loss. Sensorineural hearing loss is associated with dysfunction of the outer hair cells $(\mathrm{OHCs})$ that are involved in the active mechanism that generates the compressive on-frequency response (Ruggero and Rich, 1991; Yates, 1995; Ruggero et al., 1997). It has generally been assumed 
that the off-frequency response is not affected by the active mechanism. The finding that off-frequency TMCs are affected by hearing impairment suggests two possible explanations. First, that the post-cochlear decay of forward masking with time is slower for hearing-impaired listeners than for normal-hearing listeners. Second, that the off-frequency response is compressive in normal-hearing listeners and that damage to the active mechanism causes this response to become more linear, reducing the slope of the TMC. LopezPoveda and Alves-Pinto (2008) investigated the latter hypothesis by measuring TMCs for signal levels of 9 and $15 \mathrm{~dB}$ SL. It was assumed that, if the system were linear, the TMCs would be shifted upwards by $6 \mathrm{~dB}$. This was exactly what the authors found for a signal frequency of $4000 \mathrm{~Hz}$ and a masker frequency of $1600 \mathrm{~Hz}$. However there was evidence for a greater shift at a masker frequency of $2200 \mathrm{~Hz}$, corresponding to as much as $2: 1$ compression. If this compression is lost due to hearing impairment, this could account for the effects of hearing impairment on the off-frequency TMCs reported by Plack et al. (2004), since these authors also used a $2200-\mathrm{Hz}$ off-frequency masker.

The results of Lopez-Poveda and Alves-Pinto (2008) suggest that Plack et al. and other authors (Plack and Drga, 2003; Nelson and Schroder, 2004; Rosengard et al., 2005) may have underestimated on-frequency compression for normal-hearing listeners by using an off-frequency reference masker too close to the signal frequency. Furthermore, as acknowledged by Lopez-Poveda and Alves-Pinto, the new TMC technique depends on the assumption that the response to the signal is linear over the range of levels tested. If the signal is compressed, the technique will underestimate the compression of the maskers. Some authors have reported that such low-level signals are compressed by as much as 2:1 (Lopez-Poveda et al., 2003; Nelson and Schroder, 2004), although others have reported a more linear response (Oxenham and Plack, 1997; Nelson et al., 2001; Yasin and Plack, 2003; Plack and Skeels, 2007).

A behavioral technique for measuring compression that does not depend on the assumption of a linear off-frequency response, nor on the assumption of low-level linearity, is the additivity of nonsimultaneous masking technique, which can involve one forward and one backward masker (Oxenham and Moore, 1995), or two forward maskers (Plack and O'Hanlon, 2003; Plack et al., 2006; Plack et al., 2007; Plack et al., 2008). In the original additivity of forward masking (AFM) technique, signal threshold is measured in the presence of two temporally non-overlapping forward maskers and compared to the signal threshold for each masker presented individually. Compression, as applied to the signal, will influence the amount by which the signal level at threshold increases when the effects of the two maskers are combined: The greater the compression, the more the physical signal level has to increase to produce the same change in internal excitation. There is evidence that the internal effects of the two maskers add linearly (Plack et al., 2006; Plack et al., 2007). Based on this assumption, the increase in threshold in the combined case can be used to estimate auditory compression.
The original "signal-vary" AFM technique can only measure the on-frequency response function, since the technique estimates the compression applied to the signal. It is assumed that the signal is detected using the response of the place on the BM with $\mathrm{CF}$ close to the signal frequency. The present paper introduces a novel version of the AFM technique in which the effects of combining forward maskers on the masker levels required to mask a fixed-level signal are measured. This "masker-vary" procedure estimates the compression applied to the maskers and hence the BM response can be estimated for any frequency relative to CF. Furthermore, a range of masker threshold levels can be measured by fixing the signal at a low level and varying masker-signal interval, as in the TMC technique. This minimizes the spread of excitation of the signal on the BM, and also avoids the basalward shift in the peak of BM excitation with increases in signal level (Robles et al., 1986; Ruggero et al., 1997). The new AFM technique was used to test the hypothesis that the off-frequency BM response is linear in the base of the human cochlea.

Another aim of the present experiments was to investigate the effects of the medial olivocochlear reflex (MOCR) on estimates of the BM response. A neural feedback loop exists between the cochlea and the superior olivary complex via efferent neurons of the medial olivocochlear bundle which innervate the OHCs directly (Warr and Guinan, 1979; Liberman and Brown, 1986). Activation of the MOCR by prior acoustic stimulation reduces the gain of the OHCs (Liberman et al., 1996; Cooper and Guinan, 2006). It has been suggested that at least part of the effect of combining maskers in the AFM technique could be a consequence of gain reduction and linearization of the BM response by the MOCR triggered by the first masker (Krull and Strickland, 2008; Jennings et al., 2009). Indeed, Jennings et al. were able to simulate previous AFM results (Plack et al., 2006) using a gain-reduction model. If the MOCR produces a substantial modification of the BM response then this could affect the estimates of BM compression, and invalidate an assumption of the AFM technique that the response function does not change across the masking conditions.

To investigate the possible role of the MOCR, AFM was measured using two different durations of the first masker, $200 \mathrm{~ms}$ and $20 \mathrm{~ms}$. The second masker and signal both had a duration of $10 \mathrm{~ms}$ and the maskers and signal were presented contiguously in some conditions, so that the total duration of the stimulus was only $40 \mathrm{~ms}$ for the 20 -ms first masker in the combined condition. Backus and Guinan (2006) estimated the time course of the human MOCR using suppression of stimulus-frequency otoacoustic emissions (OAEs). They found that, after an initial delay of approximately $25 \mathrm{~ms}$, the MOCR built up relatively slowly after the onset of the elicitor. The build up could be modeled by a sum of exponentials, the fastest of which had a time constant of about $70 \mathrm{~ms}$. There was no systematic dependence of the time course on elicitor laterality. In another study on humans, James et al. (2005) reported a latency of $45 \mathrm{~ms}$ after elicitor onset for the onset of contralateral suppression of distortion product OAEs. Hence, it is reasonable to assume that the AFM conditions with a 20-ms first masker would be much less influ-

C. J. Plack and D. Arifianto: On- and off-frequency compression 
enced by the MOCR than the conditions with a 200-ms first masker. Any difference in the estimated response functions for the two first-masker durations would suggest an influence of the MOCR. If there were no differences between the response functions, this would suggest that estimates of compression using the AFM technique are not affected by the MOCR.

\section{GENERAL METHOD}

\section{A. Stimuli}

The sinusoidal signal had a frequency $\left(f_{s}\right)$ of either 4000 or $8000 \mathrm{~Hz}$. The signal had a total duration of $10 \mathrm{~ms}$, which consisted of 5-ms raised-cosine onset and offset ramps (no steady state). The maskers were 500-Hz-wide bands of noise (generated with quasi-infinite slopes by summing sinusoids with identical amplitude and random phases), with center frequencies of $f_{s}$ (on-frequency), $0.5 f_{s}$ (off-frequency), and $0.375 f_{s}$ (off-frequency). Masker 1 (M1) had a total duration of $200 \mathrm{~ms}$ (including 5-ms onset and offset ramps and 190-ms steady state) or $20 \mathrm{~ms}$ (including 5-ms onset and offset ramps and 10-ms steady state). Masker 2 (M2) had a total duration of 10 or $20 \mathrm{~ms}$, including 5-ms onset and offset ramps. The offset of M1 coincided with the onset of M2. When one or other masker was not present it was replaced by silence of the same duration, so that the temporal relations between the remaining stimuli remained the same. M1 and M2 always had the same frequency for each maskerfrequency condition (in other words, when two maskers were present they were always of the same frequency, and thresholds were compared to single maskers of the same frequency).

The experiment was run using custom-made MATLAB software on an Apple Macintosh workstation located outside a double-walled sound-attenuating booth. Stimuli were generated digitally and were output using an M-Audio 24-bit Firewire soundcard set at a clocking rate of $48 \mathrm{kHz}$. The soundcard included an anti-aliasing filter. The headphone output of the soundcard was fed via a patch-panel in the sound booth wall, without filtering or amplification, to Sennheiser HD 580 circumaural headphones. All stimuli were presented to the right ear. Listeners viewed a computer monitor through a window in the sound booth. Lights on the monitor display flashed on and off concurrently with each stimulus presentation and provided feedback at the end of each trial. Responses were recorded via a computer keyboard.

\section{B. Procedure}

A three-interval, three-alternative, forced-choice adaptive tracking procedure was used, with a 500-ms interstimulus interval. In the masking conditions, all three intervals contained either one or both maskers. One of the intervals (chosen at random) contained the signal. Threshold was determined using a two-up one-down (masker thresholds) or a two-down one-up (signal thresholds) adaptive procedure that tracked the 70.7 percent correct point on the psychometric function (Levitt, 1971). The step-size was $4 \mathrm{~dB}$ up to the fourth reversal, and was reduced to $2 \mathrm{~dB}$ for 12 sub- sequent reversals. The mean level at the last 12 reversals was taken as the threshold estimate for each block of trials. Five estimates were made for each condition and the results averaged.

First, the absolute threshold for the signal in the absence of maskers was determined. The main experiment was then conducted in two phases. Phase 1 determined levels for the individual maskers so that they were equally effective in masking the signal. Phase 2 measured the effects of combining these maskers. To control for any bias due to practice effects, some of the conditions with individual maskers were repeated between the two phases.

\section{Phase $1, M 1=M 2$}

In phase 1, depending on the experiment, the signal was presented at a range of sensation levels (with a 0-ms silent interval between M2 and the signal) or at a range of M2signal intervals (with a 10-dB signal sensation level). The range of levels or intervals was chosen separately for each listener and each frequency, limited by the need to avoid clipping when the masker level approached the maximum output of the apparatus (104 dB SPL rms), and to avoid discomfort for listeners if levels became uncomfortably loud. If one or more of the five threshold estimates for a condition was $100 \mathrm{~dB}$ SPL or higher, the data were discarded for that condition and no measurements made for that condition in phase 2. At each level or interval, the signal was presented with M1 alone, M2 alone, or M1 and M2 combined (M1 +M2). In the combined condition, the maskers had the same level. The masker level was varied adaptively to determine the level required to mask the signal. In this way, phase 1 generated pairs of roughly equally effective maskers for each signal level.

The combined condition was included so that the listener would have the same effective exposure to this stimulus as to the single-masker stimuli, hence eliminating a potential bias due to practice effects. The inclusion of the combined condition also allowed an additional measure of the BM response. Equally effective maskers provide a more sensitive measure of compression when the system is less compressive, since if one masker is more effective than the other it tends to dominate in the combined condition. However, equal-level maskers can produce an accurate estimate of compression when the system is highly compressive over the range of signal levels (for the signal-vary conditions) and masker levels (for the masker-vary conditions). In these situations, even if one masker dominates the masking, a small additional contribution from the less-effective masker will be reflected in a substantial change in physical threshold level because of the need to overcome the compression.

\section{Phase 2, Eq. Eff}

In phase 2, for each pair of equally effective maskers derived from phase 1 , the signal was presented in the presence of M1 alone, M2 alone, or M1 and M2 combined. For the signal-vary conditions, the masker levels were fixed and the signal level was varied adaptively to determine threshold. This was the procedure used by Plack and O’Hanlon (2003). 
Phase 1

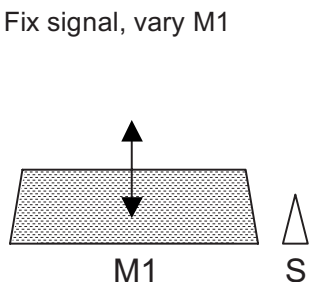

Phase 2 (signal vary)

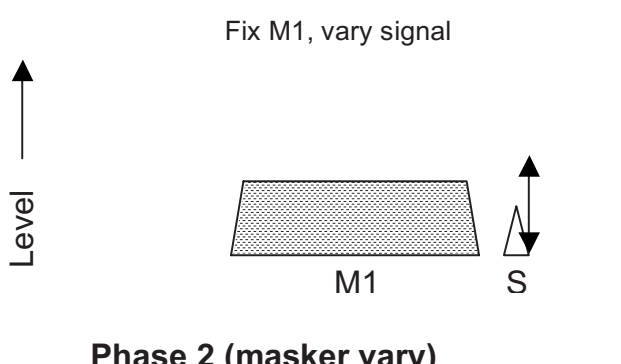

Fix signal, vary M1

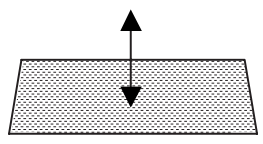

M1
Fix signal, vary $\mathrm{M} 2$

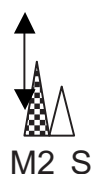

Fix M2, vary signal

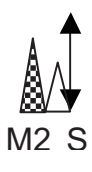

Fix signal, vary M2

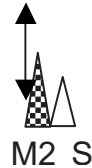

Fix signal, vary M1 and M2 together (M1 and $\mathrm{M} 2$ have equal SPLs)

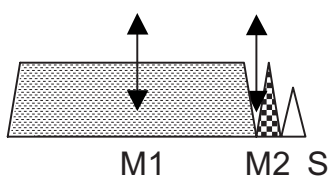

Fix M1 and M2 (M1 and M2 have equally effective levels), vary signal

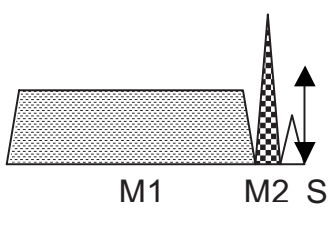

Fix signal, vary M1 and M2 together (M1 and M2 have equally effective levels)

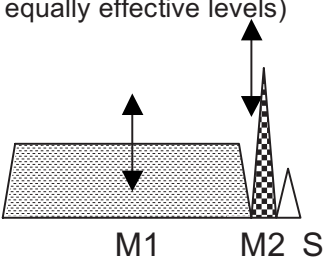

Time

FIG. 1. A schematic illustration of the procedures used in the different phases of the experiments. The arrows indicate the stimulus that was adaptively varied to determine threshold in each case. In phase 2, the level difference between the maskers in the combined conditions was fixed at the level difference between the maskers at threshold in the single masker conditions determined in phase 1 (in the illustration, it is assumed that the M2 level at threshold was higher than the M1 level at threshold). In other words, the maskers were roughly equally effective.

For the masker-vary conditions, the signal level was fixed and the masker levels were varied to determine threshold (as in phase 1). For the combined-masker conditions, the levels of the two maskers were varied together, keeping the relative level constant (at the value determined in phase 1). For the masker-vary conditions, the only difference between phase 1 and phase 2 was that for the former the maskers were presented at equal levels in the combined-masker conditions, whereas for the latter the maskers were presented at equally effective levels in the combined-masker conditions. The single masker-vary conditions were re-measured in phase 2 (randomized with the combined-masker conditions) for experimental control; to reduce the possibility that order or practice effects would affect the estimates of compression. The same fixed signal levels were used in phases 1 and 2 for the masker-vary conditions. Figure 1 is a schematic illustration of the procedure used in each phase.

Within each experiment, different masker frequencies and experiment phases were tested in different sessions. Within a session, each condition was presented once in a random order in each block of trials. When two M1 durations were used, the two M1 durations were tested in the same block. For example, in experiment 1, phase 1, five masker conditions were measured in each block for each signal level. These were: M1 (long) alone; M1 (short) alone; M2 alone; $\mathrm{M} 1$ (long) + M2; and M1(short) + M2 .

\section{ANALYSIS}

\section{A. Signal-vary conditions}

Response functions, representing the auditory processing prior to summation of the masking effects, were derived from the results using the procedure described by Plack et al. (2006) and by Plack et al. (2008). Each response function was modeled by a 3rd-order polynomial in $\mathrm{dB} / \mathrm{dB}$ coordinates, with three parameters $\left(y=a x^{3}+b x^{2}+c x\right)$. In units of intensity, this becomes:

$$
f(x)=10^{\left(a .\left(10 \log _{10}(x)\right)^{3}+b \cdot\left(10 \log _{10}(x)\right)^{2}+c .10 \log _{10}(x)\right) / 10,}
$$

where $x$ is input intensity, and $a, b$, and $c$ are the coefficients of the polynomial. (The constant or intercept in the polynomial is not constrained by the data and does not affect the predictions of the model.) A separate polynomial was derived for each listener.

After preprocessing by the response function, $f$, the responses to the stimuli (maskers and signal) were assumed to

C. J. Plack and D. Arifianto: On- and off-frequency compression 
add linearly. Detection of the signal was based on the signalto-masker ratio after preprocessing and summation, and this ratio was assumed to be constant at threshold for all conditions:

$$
E=f(S),
$$

where $S$ is the signal intensity at threshold and $E$ is described as the "masking effect," a measure of the amount of masking of the signal, defined by the internal excitation (in units of intensity) of the signal at threshold (i.e., after processing by the function $f$ ). Assuming that the effects of two maskers sum linearly:

$$
E_{\mathrm{M} 1+\mathrm{M} 2}=E_{\mathrm{M} 1}+E_{\mathrm{M} 2},
$$

where $E_{\mathrm{M} 1}, E_{\mathrm{M} 2}$, and $E_{\mathrm{M} 1+\mathrm{M} 2}$ are the masking effects produced by M1, M2, and M1 and M2 combined. Substituting from Eq. (2) and solving for $S$ gives:

$$
S_{\mathrm{M} 1+\mathrm{M} 2}=f^{-1}\left(f\left(S_{\mathrm{M} 1}\right)+f\left(S_{\mathrm{M} 2}\right)\right) \text {, }
$$

where $S_{\mathrm{M} 1}$ and $S_{\mathrm{M} 2}$ are the signal intensities at threshold in the presence of $\mathrm{M} 1$ and $\mathrm{M} 2$, respectively, and $S_{\mathrm{M} 1+\mathrm{M} 2}$ is the signal intensity at threshold in the presence of M1 and M2 combined. Using this equation, and Eq. (1) as the function, $f$, the thresholds from each masker alone in phase $2\left(S_{\mathrm{M} 1}\right.$ and $S_{\mathrm{M} 2}$ ) were used as the input to the model and the thresholds in the presence of both maskers $\left(S_{\mathrm{M} 1+\mathrm{M} 2}\right)$ in phase 2 were predicted. For each listener independently, the coefficients of Eq. (1) $(a, b$, and $c)$ were selected to minimize the sum of the squared deviations of the model predictions from the thresholds (expressed in $\mathrm{dB}$ ) in the combined-masker conditions.

\section{B. Masker-vary conditions}

The procedure for deriving response functions from the masker-vary data is based on the same assumptions as those used for the signal-vary data. The masking effect $E$ is equal to the masker intensity after preprocessing by the response function multiplied by a constant that is invariant with masker level:

$$
E=k f(M),
$$

where $k$ is a dimensionless constant that takes into account the effects of stimulus parameters including masker duration, masker frequency, and masker-signal interval. Since the signal level is constant in the masker-vary conditions:

$$
\begin{aligned}
& E=k_{1} f\left(M_{1}\right), \\
& E=k_{2} f\left(M_{2}\right),
\end{aligned}
$$

where $M_{1}$ and $M_{2}$ are the masker intensities at threshold in the single-masker conditions, and $k_{1}$ and $k_{2}$ are dimensionless constants determined by the stimulus parameters of the two maskers. Assuming the masking effects sum linearly in the combined conditions:

$$
E=k_{1} f\left(M_{1 a}\right)+k_{2} f\left(M_{2 a}\right),
$$

where $M_{1 \mathrm{a}}$ and $M_{2 \mathrm{a}}$ are the intensities of M1 and M2 at threshold in the combined-masker conditions. Substituting for $k_{1}$ and $k_{2}$ in Eq. (8) and dividing by $E$ gives:

$$
\begin{aligned}
& 1=\frac{f\left(M_{1 a}\right)}{f\left(M_{1}\right)}+\frac{f\left(M_{2 a}\right)}{f\left(M_{2}\right)}, \\
& M_{1 a}=f^{-1}\left(f\left(M_{1}\right)\left(1-\frac{f\left(M_{2 a}\right)}{f\left(M_{2}\right)}\right)\right) .
\end{aligned}
$$

Using this equation, and Eq. (1) as the function $f$, the masker intensities at threshold in the single masker conditions, and the masker intensities at threshold for M2 in the combined masker conditions, were used to predict the intensities of M1 at threshold in the combined masker conditions. For each listener independently, the coefficients of Eq. (1) ( $a, b$, and $c$ ) were selected to minimize the sum of the squared deviations of the model predictions from the thresholds for M1 (expressed in $\mathrm{dB}$ ) in the combined-masker conditions.

Unlike previous AFM experiments, combined-masker conditions were tested in phase 1 in all but one of the present experiments. Hence, response functions could have been derived from the results of phase 1 alone. However, because the maskers in the combined conditions in phase 1 were equal-level rather than equally effective, in some cases one of the two maskers dominated the masking. In these cases, the procedure for deriving response functions is unreliable: When the level difference between the dominant singlemasker condition and the combined condition is small, a small change in threshold can produce a very large change in the estimated compression exponent (see Plack and O'Hanlon, 2003). To make full use of the phase 1 results, response functions were fit to the phase 1 and phase 2 data combined.

\section{EXPERIMENT 1: 4/2 KHZ, SIGNAL- AND MASKER- VARY, SENSATION LEVEL}

\section{A. Rationale}

There were three aims for the first experiment. First, to validate the new masker-vary AFM technique by comparing the results from this technique with those of the original signal-vary technique for the same on-frequency conditions. Second, to investigate compression for off-frequency maskers. Third, to investigate the potential effects of MOCR activation on derived response functions. To this end, two durations of M1, $200 \mathrm{~ms}$ and $20 \mathrm{~ms}$, were tested. It was assumed that the former would produce greater MOCR activation than the latter.

\section{B. Conditions}

The signal frequency was $4000 \mathrm{~Hz}$. For the signal-vary technique, on-frequency maskers were used. For the maskervary technique, on- and off-frequency $\left(0.5 f_{s}\right)$ maskers were used. M1 duration was either 200 or $20 \mathrm{~ms}$ for the onfrequency condition, and $200 \mathrm{~ms}$ for the off-frequency condition. M2 duration was $10 \mathrm{~ms}$. The M2-signal silent interval was $0 \mathrm{~ms}$. Measurements were made for a range of signal sensation levels. 


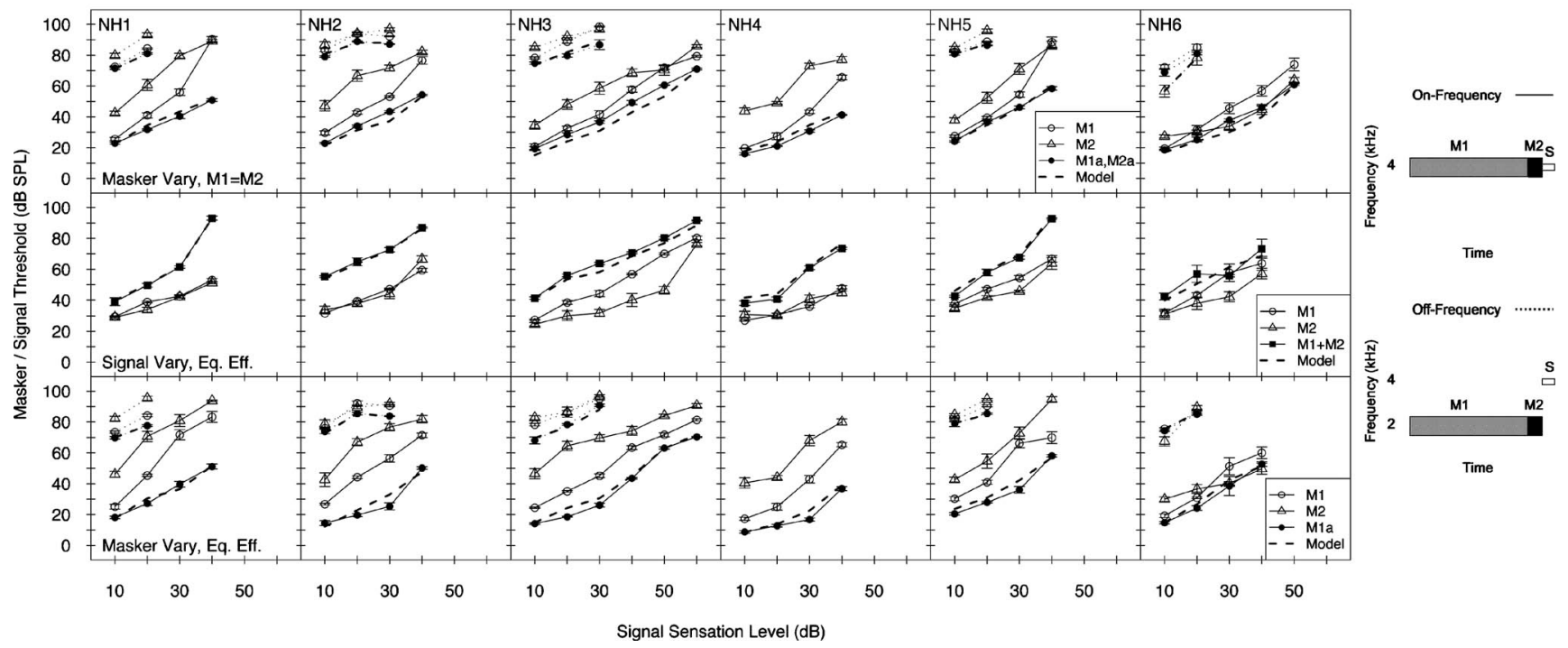

FIG. 2. The individual results of experiment 1 for the 200-ms M1 conditions. Error bars show standard errors. The top panels show the masker levels at threshold for phase 1 of the experiment. Open symbols show the results for the individual masker conditions, filled symbols show the results for the combined masker conditions (in which the maskers had the same level). Results for on-frequency $(4000-\mathrm{Hz})$ maskers are connected by continuous lines, results for off-frequency $(2000-\mathrm{Hz})$ maskers are connected by dotted lines. The middle panels show the signal thresholds measured in phase 2 in the presence of equally effective masker levels. For these thresholds, the $x$-axis shows the nominal sensation level (i.e., the sensation level used in phase 1 to determine the equally effective masker levels). The bottom panels show the masker thresholds measured in phase 2 with maskers set to equally effective levels in the combined case. Only the level of M1a is shown. Bold dashed lines show the predictions of the model described in the text. Schematic spectrograms of the stimuli are provided on the right.

\section{Listeners}

Six normal-hearing listeners were tested (absolute thresholds were less than $20 \mathrm{~dB} \mathrm{HL}$ at all frequencies tested). Ages ranged from 24 to 35 years (mean 31.3). One of the listeners (NH4) was not tested on the off-frequency conditions. All listeners were given several hours training on the task before data collection began. Thresholds for the 10-ms signal in quiet were $22,22,21,19,25$, and $20 \mathrm{~dB}$ SPL for $\mathrm{NH} 1, \mathrm{NH} 2, \mathrm{NH} 3, \mathrm{NH} 4, \mathrm{NH} 5$, and $\mathrm{NH} 6$ respectively.

\section{Results}

The results for phases 1 and 2 are presented in Fig. 2 (M1=200 ms) and Fig. 3 (M1=20 ms). Open symbols show the results for the individual maskers and the filled symbols show the results for the combined maskers. Onfrequency thresholds are connected by continuous lines, and off-frequency thresholds are connected by dotted lines. Note that the thresholds for M2 alone are identical in the two

Signal Sensation Level, $4 \mathrm{kHz}, \mathrm{M} 1=20 \mathrm{~ms}$

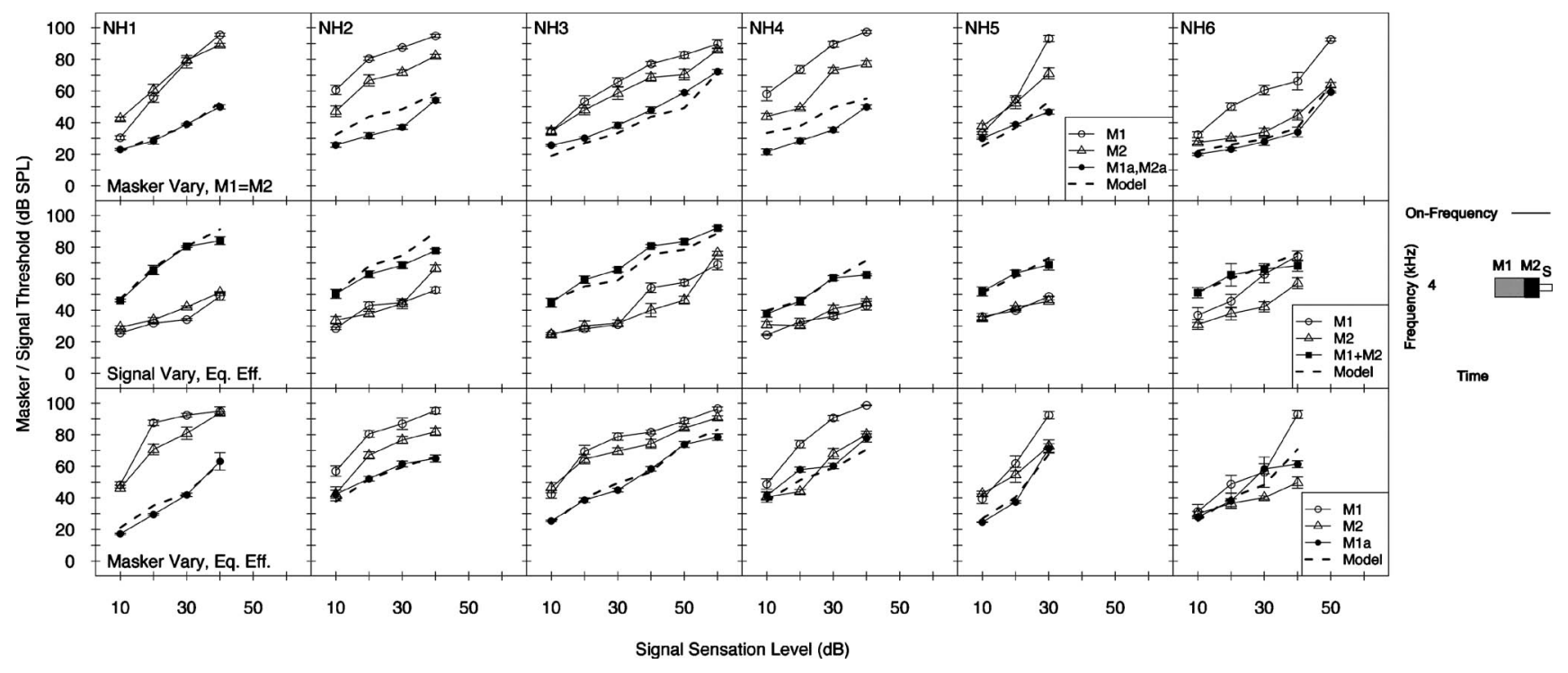

FIG. 3. The individual results of experiment 1 for the 20-ms M1 conditions. The meanings of symbols are the same as for Fig. 2. 


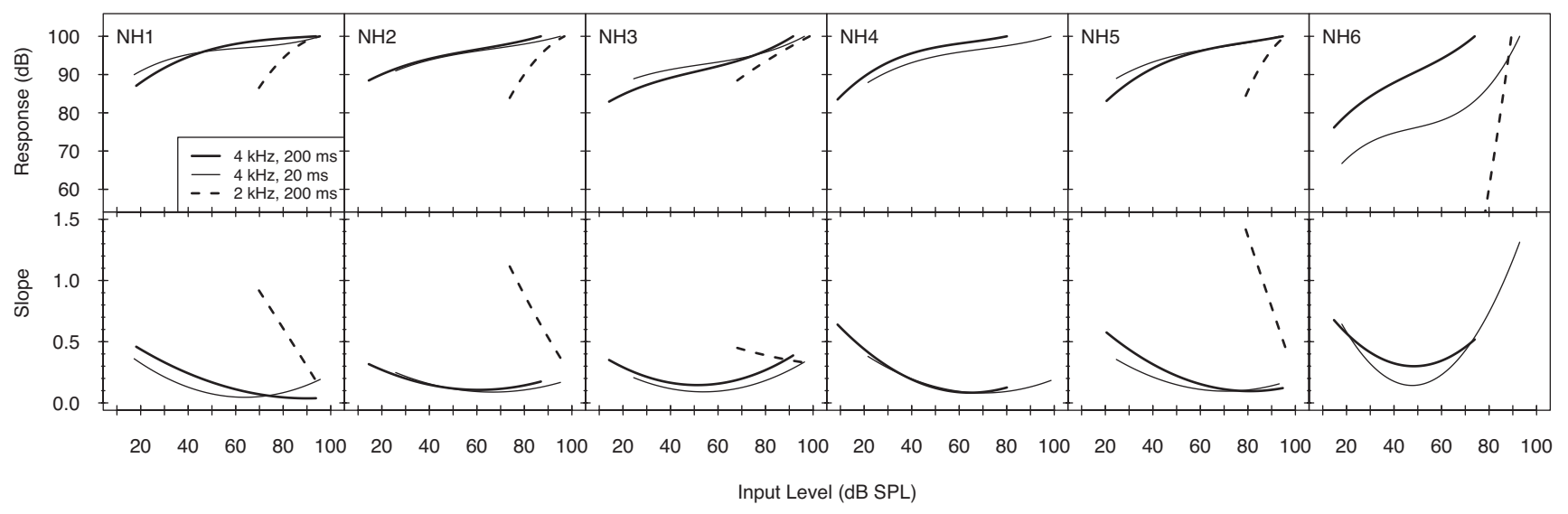

FIG. 4. Response functions (top panels) and response-function slopes (bottom panels) derived from the data of experiment 1 .

figures. The M2 alone conditions were identical for the two M1 duration conditions, and were only measured once.

The top panels show the masker thresholds from phase 1 , in which the signal was presented at a range of sensation levels and masker level was varied to obtain threshold. In the combined case, the two maskers had the same SPL. The middle panels show the results for the phase 2 signal-vary conditions, in which the masker levels were fixed at the levels determined in phase 1 , and the threshold of the signal determined. This is the standard AFM procedure that has been used in previous studies. In an intensity-linear system, equally effective maskers should produre a $3-\mathrm{dB}$ increase in signal threshold compared to the individual-masker conditions. The differences between the combined and individualmasker conditions are generally much greater than this, which is indicative of strong compression.

The signal-vary phase 2 results show that phase 1 was not always successful in generating equally effective maskers. In particular, for NH3 for the 200-ms M1, and for NH6 for the 20-ms M1, signal thresholds are different for the two maskers that were supposedly equally effective (compare open circles and triangles, middle panels). This may reflect the effects of practice. However, since the fitting procedure uses the threshold values for each condition rather than assuming equal effectiveness, these anomalies should not affect the reliability of the fits.

The bottom panels show the results for the phase 2 masker-vary conditions in which the signal was presented at a range of sensation levels, and masker level was varied to obtain threshold. In the combined conditions, the difference between the masker levels was fixed at the threshold difference for the individual maskers measured in phase 1. The masker level for M1 in the combined case (M1a) is illustrated. For the on-frequency conditions, for both M1 durations, evidence of strong compression is also seen in the masker-vary results. In an intensity-linear system, equally effective maskers should result in a 3-dB decrease in threshold when combined compared to the individual-masker conditions. The differences between the combined and individual masker conditions are generally much greater than this, which is indicative of strong compression. Off- frequency thresholds for the individual and combined conditions are closer together suggesting a more linear response. However, even in these conditions there is evidence of substantial compression in some cases, for example $\mathrm{NH} 1$ and NH3. There is some evidence of an improvement in performance (presumably related to practice) between phase 1 (top panels) and phase 2 (bottom panels) for identical individualmasker conditions, in that masker thresholds are in some cases higher for the latter (for example, Fig. 2, NH5, M2 thresholds).

Response functions were derived from the data using the fitting procedure described in Section III. A single set of coefficients was derived for each listener, for each M1 duration, and each masker frequency. In other words, the phase 1 and phase 2 data (signal and masker vary) were all fitted together. For each predicted combined-masker threshold, the individual thresholds for the same specific signal level/ masker-signal interval, M1 duration, masker frequency, and phase in the experiment were used as inputs to the model. The predicted thresholds for the combined-masker conditions are shown by the bold dashed lines. In most case, the model provides a good account of the data. The mean rms errors of the fits (in $\mathrm{dB})$ are $3.2(200 \mathrm{~ms}$, on), $2.0(200 \mathrm{~ms}$, off), and $5.1(20 \mathrm{~ms}$, on). In particular, note that the same response function can account for the signal-vary and masker-vary conditions. This is good evidence that the procedures are measuring the same processes, and provides validation for the new masker-vary AFM technique. For two listeners ( $\mathrm{NH} 2$ and $\mathrm{NH} 4)$ for $\mathrm{M} 1=20 \mathrm{~ms}$, the model overestimates the combined thresholds in phase 1 , but provides a good fit to the thresholds in phase 2 .

The response functions are illustrated in the top panels of Fig. 4. The fitting procedure does not provide any information about the y-intercept in the polynomial (and hence does not directly measure gain), and the functions have been normalized (arbitrarily) so that the maximum output level is $100 \mathrm{~dB}$. Each function is only plotted for a range of input levels equal to the range of threshold values measured in the experiment (for each listener), since the form of the function beyond this range is not constrained by the data.

The on-frequency functions show the expected shape, 


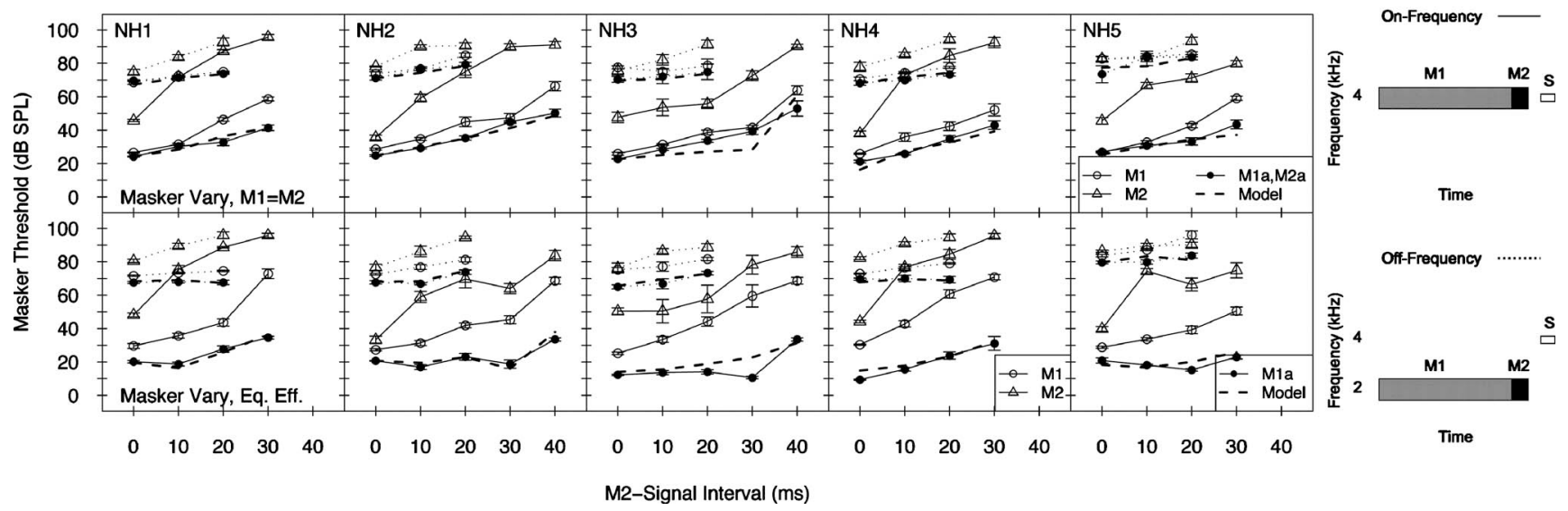

FIG. 5. The individual results of experiment 2 for the 200-ms M1 conditions. The $x$ axis shows the time interval between the offset of M2 and the onset of the signal. For the M1 alone conditions, the time interval is still measured from the offset of the absent M2. In other words, the time intervals between the offset of M1 and the onset of the signal are these x values plus the duration of M2 (10 ms). The meanings of symbols are the same as for Fig. 2.

with a highly compressive mid-level region and more linear low-level, and in some cases high-level, regions. These functions are similar to those reported previously using TMC (Nelson et al., 2001) and AFM (Plack et al., 2008) techniques. The bottom panels show the slopes of these functions. These values are equal to the compression exponents. With the exception of NH6 at high levels, all the slopes are less than unity, indicating compression across the level range. Between 40 and $80 \mathrm{~dB}$ SPL, the mean slopes for the on-frequency functions (calculated by averaging the slope values over the input range $40-80 \mathrm{~dB}$ SPL at 1 -dB spacing) are 0.18 for the $200-\mathrm{ms} \mathrm{M} 1$ and 0.14 for the 20-ms M1. A paired-samples t-test revealed that this difference is significant ( $\mathrm{p}=0.019$, two-tailed): Compression decreases as M1 duration is increased.

The off-frequency functions are all more linear than the on-frequency functions. However, for all listeners except NH6, the slope decreases as level is increased, with evidence of compression at the highest level. The off-frequency response function for NH6 is not well defined by the data, and the slope values are greater than the $y$-axis limit. When the combined and individual masker thresholds are similar, as in this case, the estimate of compression becomes highly variable, and can reach large values (Plack and O'Hanlon, 2003). Including NH6, the mean off-frequency slope across all levels defined by the data (calculated for individual listeners) is 1.22 , however the value for NH6 $(3.59,1.77$ standard deviations from the mean) clearly has a disproportionate effect on the mean. Excluding NH6, the mean is 0.64 , which a t-test revealed to be significantly less than $1(\mathrm{p}=0.026)$. The significance of this effect for individual listeners was calculated using all the off-frequency data points for phases 1 and 2 for the masker-vary conditions. The M1a thresholds $(n=4$ or 6 depending on listener) were compared to predictions based on a linear response function. Linear predictions were obtained by setting function $f$ to the identity function $[f(x)$ $=x]$ and solving Eq. (10) $\left(M_{2 \mathrm{a}}\right.$ was set at the appropriate intensity so that the intensity ratio between predicted $M_{1 \text { a }}$ and $M_{2 \mathrm{a}}$ was the same as that tested in each condition). The predicted and obtained values were compared using a one- tailed t-test. M1a thresholds are significantly lower (p $<0.05)$ than the linear predictions (indicating compression) for $\mathrm{NH} 1, \mathrm{NH} 2$, and $\mathrm{NH} 3$.

\section{EXPERIMENT 2: 4/2 KHZ, MASKER-VARY, MASKER-SIGNAL INTERVAL}

\section{A. Rationale}

Experiment 1 provided validation of the masker-vary technique, and evidence that the off-frequency BM response may be compressive at high levels for some listeners. The aim of the second experiment was to investigate this finding further using a different version of the masker-vary technique. The signal sensation level was fixed at $10 \mathrm{~dB}$, and the M2-signal silent interval was varied to produce a range of masker levels at threshold. Like the TMC technique, the use of a low-level signal in the AFM technique has the advantage of limiting the region of the BM stimulated to suprathreshold levels by the signal (Nelson et al., 2001). The use of a fixed signal level also eliminates the basalward shift of the traveling wave that occurs when signal level is increased. Excitation spread and basalward shift tend to make the estimate of the BM response more linear by allowing detection at places with CFs removed from the frequency of the signal ("offfrequency listening," Johnson-Davies and Patterson, 1979; Oxenham, 1997). Hence, it was expected that the estimated response functions would (if anything) be more compressive in experiment 2 than in experiment 1 , and provide a more accurate measure of the response at the $4000-\mathrm{Hz}$ place.

\section{B. Conditions}

The signal frequency was $4000 \mathrm{~Hz}$. On- and offfrequency $\left(0.5 f_{s}\right)$ maskers were used. M1 duration was either 200 or $20 \mathrm{~ms}$. M2 duration was $10 \mathrm{~ms}$. Signal sensation level was fixed at $10 \mathrm{~dB}$. Measurements were made for a range of M2-signal silent intervals (measured offset-onset). 


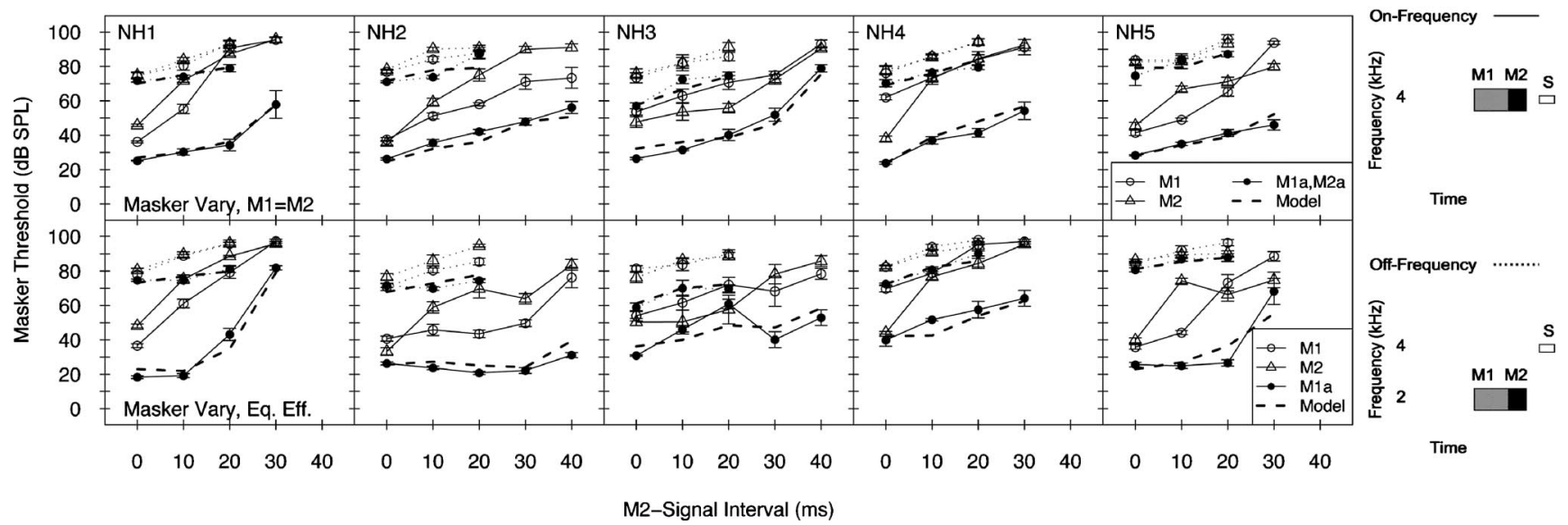

FIG. 6. The individual results of experiment 2 for the 20-ms M1 conditions. The meanings of symbols are the same as for Fig. 2.

\section{Listeners}

Five of the listeners who took part in experiment 1 were tested. Ages ranged from 24 to 35 years (mean 31.2). All listeners were given several hours training on the task before data collection began.

\section{Results}

The results are presented in Fig. $5(\mathrm{M} 1=200 \mathrm{~ms})$ and Fig. $6(\mathrm{M} 1=20 \mathrm{~ms})$. The phase 1 results are presented in the top panels and the phase 2 results are presented in the bottom panels. Note again that the thresholds for M2 alone are identical in the two figures. As in experiment 1, the on-frequency results show large differences between the single and combined masker conditions, indicative of compression. The offfrequency thresholds are closer together, but again there is clear evidence of compression for most listeners, particularly at the higher levels.

Response functions derived from these data are shown in Fig. 7, together with the slopes of these functions. The mean rms errors of the fits (in $\mathrm{dB}$ ) are 3.4 (200 ms, on), 1.6
(200 ms, off), 5.0 (20 ms, on), and 2.8 (20 ms, off). The on-frequency functions are similar to those in experiment 1 , except that the functions are even more compressive. Between 40 and $80 \mathrm{~dB}$ SPL, the mean slopes for the onfrequency functions are 0.08 for the $200-\mathrm{ms}$ M1 and 0.09 for the 20-ms M1. This difference is not significant. The functions are less compressive at low levels for the 200-ms M1 compared to the 20-ms M1 for three of the five listeners (NH2, NH3, and NH5).

The results for the off-frequency maskers are striking, showing substantial compression at high levels for all listeners for both masker durations. The mean slopes are 0.42 for the 200-ms M1 and 0.36 for the 20-ms M1, both significantly less than 1 ( $\mathrm{p}<0.001$ in both cases). The same individual analysis used in experiment 1 was used here. The M1a thresholds are significantly lower $(\mathrm{p}<0.05)$ than the linear predictions for NH2, NH3, and NH4 for the 200-ms M1, and for NH1, NH2, NH3, and NH4 for the 20-ms M1. These data support the findings of experiment 1 regarding off-frequency compression.

Masker-Signal Interval, 4 / 2 kHz

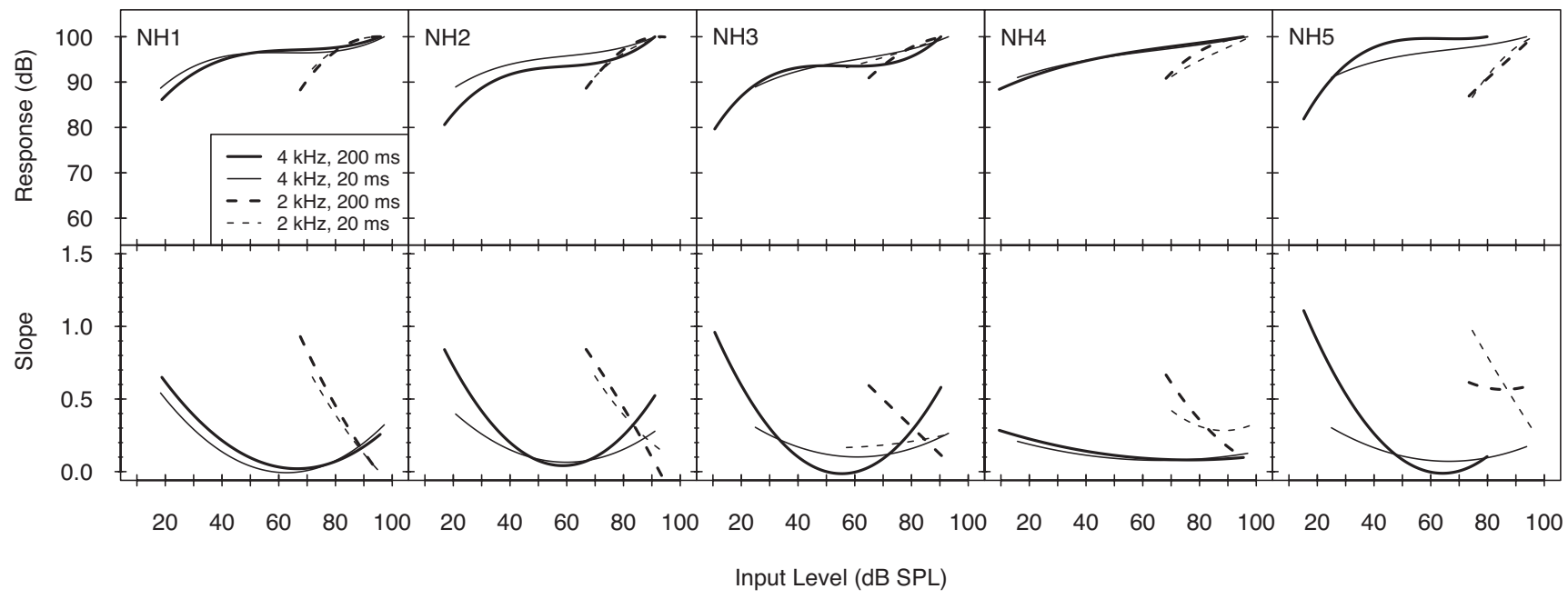

FIG. 7. Response functions (top panels) and response-function slopes (bottom panels) derived from the data of experiment 2. 


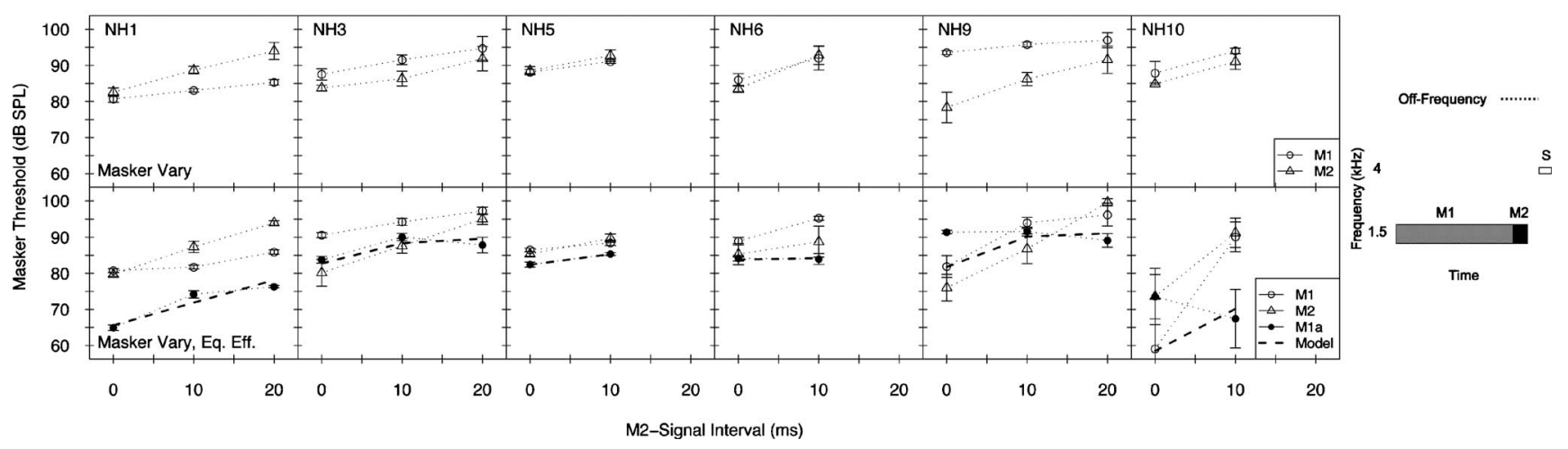

FIG. 8. The individual results of experiment 3. The meanings of symbols are the same as for Fig. 2, except that only the off-frequency (1500-Hz) masker conditions were tested, and the combined masker conditions were not tested in phase 1 (top panels).

\section{EXPERIMENT 3: 4/1.5 KHZ, MASKER-VARY, MASKER-SIGNAL INTERVAL}

\section{A. Rationale}

Experiments 1 and 2 provide evidence for off-frequency compression an octave below the signal frequency. The findings are broadly consistent with the results of Lopez-Poveda and Alves-Pinto (2008) obtained using their modified TMC technique. For a 4000-Hz signal they found evidence for compression at $2200 \mathrm{~Hz}$. However, the response to a $1600-\mathrm{Hz}$ masker was linear. To test the hypothesis that a more linear response occurs for these lower masker frequencies, the modified AFM technique was used to measure response functions with a $1500-\mathrm{Hz}$ masker.

\section{B. Conditions}

The signal frequency was $4000 \mathrm{~Hz}$. Only off-frequency $\left(0.375 f_{s}\right)$ maskers were used. M1 duration was $200 \mathrm{~ms}$ and M2 duration was $20 \mathrm{~ms}$. Signal sensation level was fixed at $10 \mathrm{~dB}$. Measurements were made for a range of M2-signal silent intervals (measured offset-onset). In phase 1 the combined masker thresholds were not measured, hence the response-function estimation is dependent on the results of phase 2 with (approximately) equally effective maskers.

\section{Listeners}

Six normal-hearing listeners took part, four of whom also participated in experiment 1. Ages ranged from 24 to 35 years (mean 32.5). All listeners were given several hours training on the task before data collection began. Thresholds for the 10-ms signal in quiet were 22, 21, 25, 20, 21, and 25 dB SPL for NH1, NH3, NH5, NH6, NH9, and NH10 respectively.

\section{Results}

The results for phases 1 and 2 are presented in Fig. 8, and the response functions derived from the data are shown in Fig. 9. The thresholds of NH9 and NH10 in phase 2 were highly variable, in some cases showing less masking for the combined-masker conditions than the individual conditions. The goodness of fit of the model was variable between listeners. The rms errors (in $\mathrm{dB}$ ) were 1.8 (NH1), 1.5 (NH3), 0.1 (NH5), 0.3 (NH6), 5.8 (NH9), and 10.8 (NH10). The results for NH5 provide little evidence of compression, but considerable compression is evident in the results for the other five listeners (Fig. 9). Four of the listeners (NH3, NH6, NH9, and NH10) show decreasing exponents with increasing level, although the other two listeners show the opposite effect. The low number of points $(n=2$ or 3$)$ meant that the

Masker-Signal Interval, 4 / $1.5 \mathrm{kHz}$

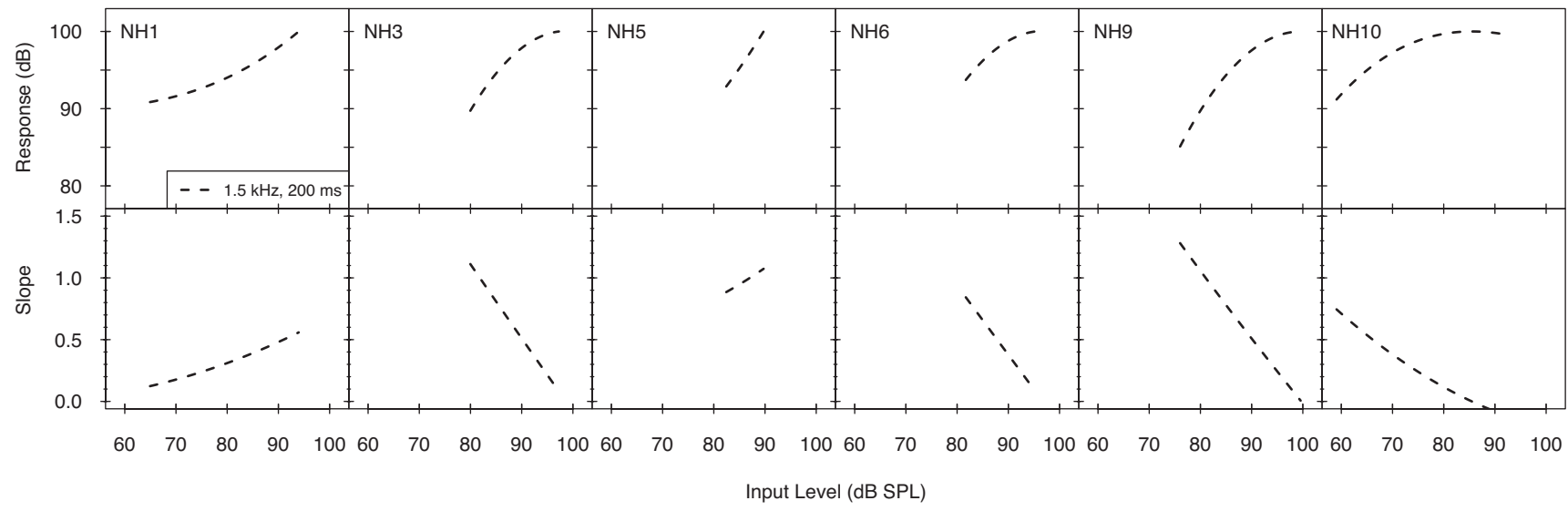

FIG. 9. Response functions (top panels) and response-function slopes (bottom panels) derived from the data of experiment 3 . 


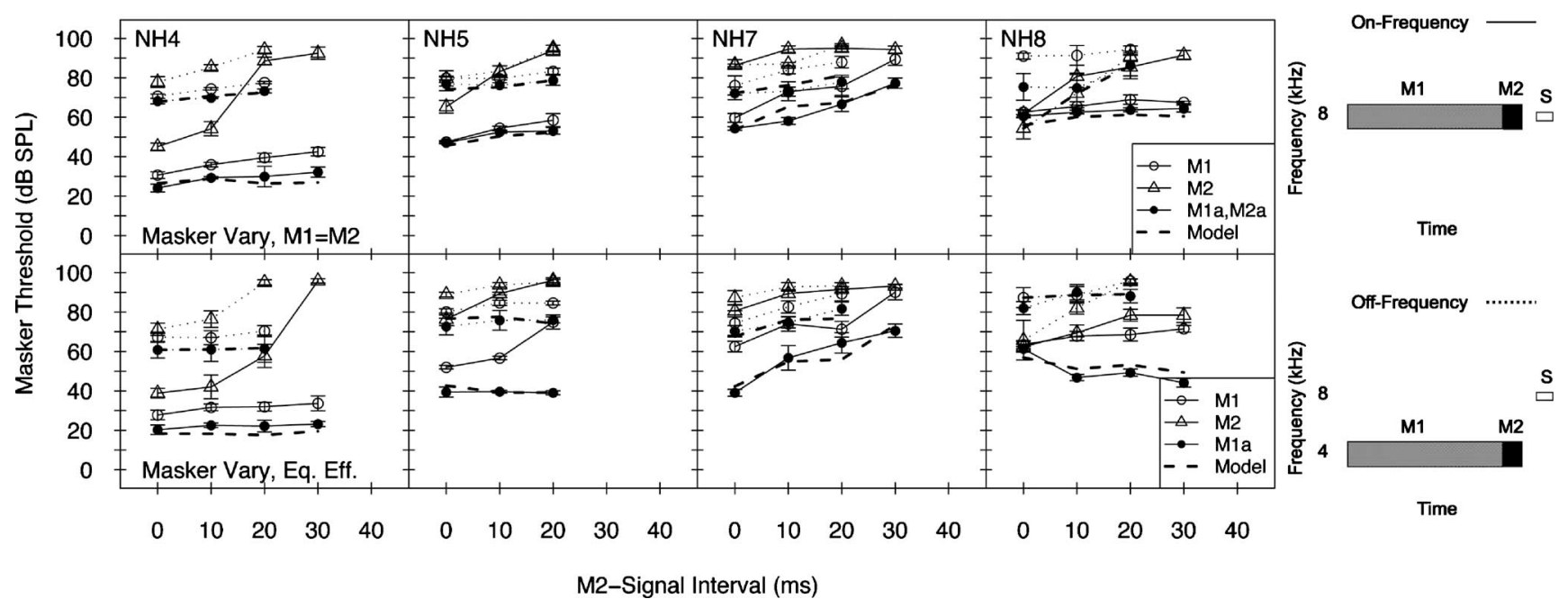

FIG. 10. The individual results of experiment 4 for the 200-ms M1 conditions. The meanings of symbols are the same as for Fig. 2, except that the on-frequency maskers had a frequency of $8000 \mathrm{~Hz}$, and the off-frequency maskers had a frequency of $4000 \mathrm{~Hz}$.

individual analyses described earlier did not reveal a significant deviation from linear predictions except for listener NH1. However, the mean slope of the off-frequency response functions is 0.54 , and a t-test revealed that this is significantly less than $1(\mathrm{p}=0.003)$. Excluding the variable results of NH9 and NH10, the mean off-frequency slope is 0.58 , and this is also significantly less than $1(\mathrm{p}=0.029)$.

\section{EXPERIMENT 4: 8/4 KHZ, MASKER-VARY, MASKER-SIGNAL INTERVAL}

\section{A. Rationale}

The results of the first three experiments suggest that the off-frequency response is compressive at the $4000-\mathrm{Hz}$ place, with mean compression estimates ranging from 0.36 to 0.64 (excluding NH6 from experiment 1). It was considered that a possible source of the compression could be the stapedius reflex arc, which has a neural pathway involving the auditory nerve, cochlear nucleus, superior olivary complex, and facial nerve, resulting in contraction of the stapedius muscle in the middle ear. Contraction of the stapedius muscle reduces transmission of sound to the cochlea. The stapedius reflex is triggered by sound levels in excess of about $85 \mathrm{~dB}$ SPL and has a low-pass characteristic, mainly affecting frequencies below $1000 \mathrm{~Hz}$, but extending up to $2000 \mathrm{~Hz}$ in humans (Rabinowitz, 1977; Rosowski and Relkin, 2001). Although the attenuation provided by the reflex near $2000 \mathrm{~Hz}$ may be less than $1 \mathrm{~dB}$, it is conceivable that the high-level offfrequency maskers were slightly attenuated by the reflex, contributing to the compressive response. This could partly explain why off-frequency compression increased with increasing level in several cases. To test this hypothesis, in the final experiment the measurements were repeated using an $8000-\mathrm{Hz}$ signal frequency and 4000-Hz masker frequency, so that the off-frequency response would be unaffected by the reflex. If off-frequency compression were dependent on the stapedius reflex, then the off-frequency response at $8000 \mathrm{~Hz}$ should be less compressive than that at $4000 \mathrm{~Hz}$.

\section{B. Conditions}

The signal frequency was $8000 \mathrm{~Hz}$. On- and offfrequency $\left(0.5 f_{s}\right)$ maskers were used. M1 duration was either 200 or 20 ms. M2 duration was 10 ms. Signal sensation level was fixed at $10 \mathrm{~dB}$. Measurements were made for a range of M2-signal silent intervals (measured offset-onset).

\section{Listeners}

Four normal-hearing listeners took part, two of whom had participated in experiment 1. Ages ranged from 24 to 34 years (mean 30.8). All listeners were given several hours training on the task before data collection began. Thresholds for the 10-ms signal in quiet were 26, 34, 31, and $35 \mathrm{~dB}$ SPL for $\mathrm{NH} 4, \mathrm{NH} 5, \mathrm{NH} 7$, and $\mathrm{NH} 8$ respectively.

\section{Results}

The results for phases 1 and 2 are presented in Fig. 10 $(\mathrm{M} 1=200 \mathrm{~ms})$ and Fig. $11(\mathrm{M} 1=20 \mathrm{~ms})$. Note again that the thresholds for M2 alone are identical in the two figures. Consistent with the results of experiments 1 and 2, the onfrequency results show large differences between the single and combined masker conditions, indicative of compression. The results for NH8 for the 200-ms M1 appear anomalous, as the M1a values decrease with increasing masker-signal interval. However, a comparison with the phase 1 results (upper panel) suggests the explanation. In phase 1, the M2 threshold was much higher than the M1 threshold, so that in the (nominally) equally effective combined masker used in phase 2, the M2 level was set over $20 \mathrm{~dB}$ higher than the M1 level for the larger intervals. However, the individual-masker thresholds measured in phase 2 (which are replications of the individual-masker conditions in phase 1) are much closer together. This means that masking in the combined-masker conditions would have been largely dominated by M2. The M2a threshold (not illustrated) increases from 60 to $68 \mathrm{~dB}$ 


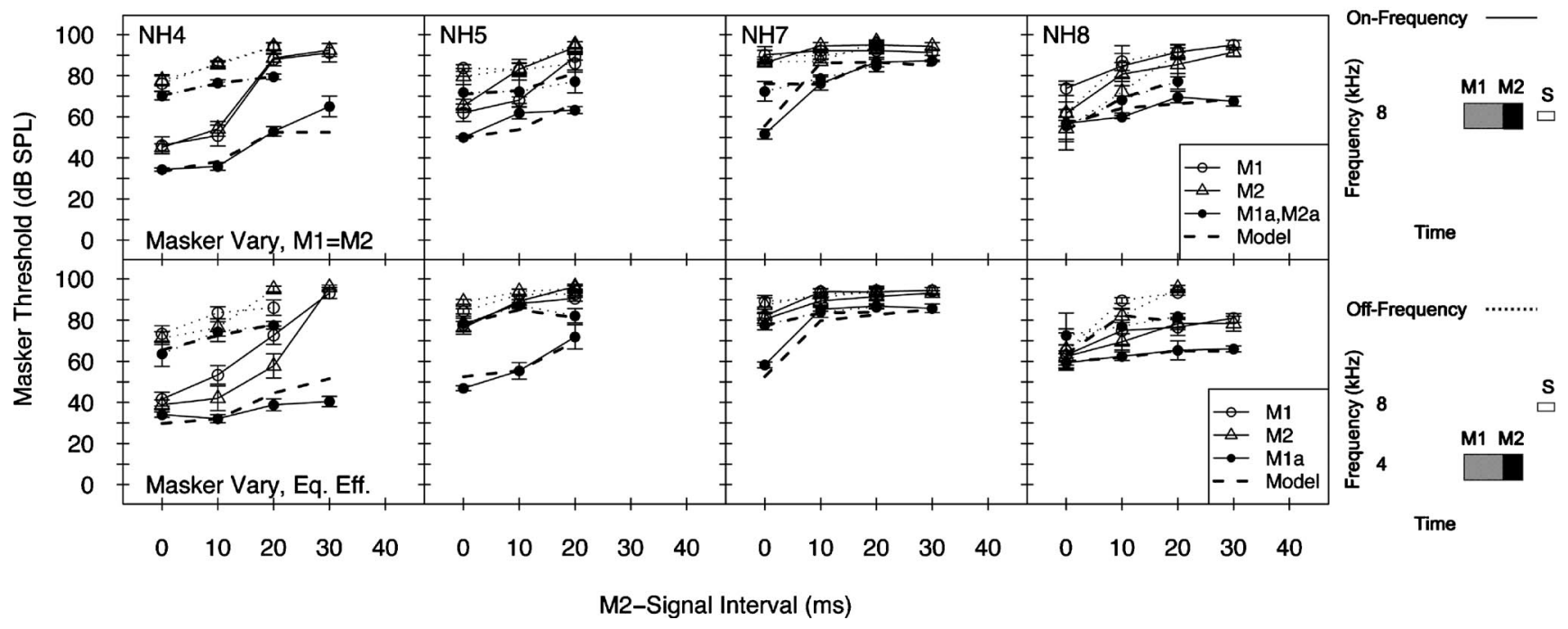

FIG. 11. The individual results of experiment 4 for the 20-ms M1 conditions. The meanings of symbols are the same as for Fig. 2, except that the on-frequency maskers had a frequency of $8000 \mathrm{~Hz}$, and the off-frequency maskers had a frequency of $4000 \mathrm{~Hz}$.

SPL over the four masker-signal intervals, but the M1a level, being set an increasingly negative relative level, is seen to decrease.

The off-frequency thresholds are closer together, but again there is clear evidence of compression for most listeners, particularly at the higher levels. For three of the four listeners, the off-frequency thresholds are closer to the onfrequency thresholds than in experiments 1 and 2 .

Response functions are shown in Fig. 12. The mean rms errors of the fits (in $\mathrm{dB})$ are $2.9(200 \mathrm{~ms}$, on), $3.7(200 \mathrm{~ms}$, off), 4.5 (20 ms, on), and 2.5 (20 ms, off). Between 50 and $90 \mathrm{~dB}$ SPL, the mean slopes for the on-frequency functions are 0.13 for the $200-\mathrm{ms}$ M1 and 0.16 for the $20-\mathrm{ms} \mathrm{M} 1$. This difference is not significant. The mean slopes for the offfrequency functions are 0.58 for the $200-\mathrm{ms}$ M1 and 0.34 for the 20-ms M1, although only the latter is significantly less than $1(\mathrm{p}<0.001)$. Individual analyses revealed that the M1a thresholds are significantly lower $(\mathrm{p}<0.05)$ than the linear predictions for $\mathrm{NH} 4, \mathrm{NH} 5$, and $\mathrm{NH} 7$ for both $\mathrm{M} 1$ durations.

\section{DISCUSSION}

\section{A. The masker-vary AFM technique}

This study used a new version of the AFM technique that measures the effect of combining two maskers on the masker level required to mask a signal. The masker-vary technique presumably estimates compression of the masker at the signal place. In experiment 1 the new technique was compared with the original signal-vary AFM technique in which signal threshold is measured to estimate compression of the signal at the signal place. For the on-frequency conditions, the two techniques produced thresholds that are compatible with the same response function for each individual, suggesting that the techniques are measuring the same underlying processes.

Estimates of the compression exponent for the onfrequency maskers are at the lower end of the estimates reported previously. The mean compression exponents for the sensation-level conditions (experiment 1) are 0.18 for the

Masker-Signal Interval, 8 / 4 kHz

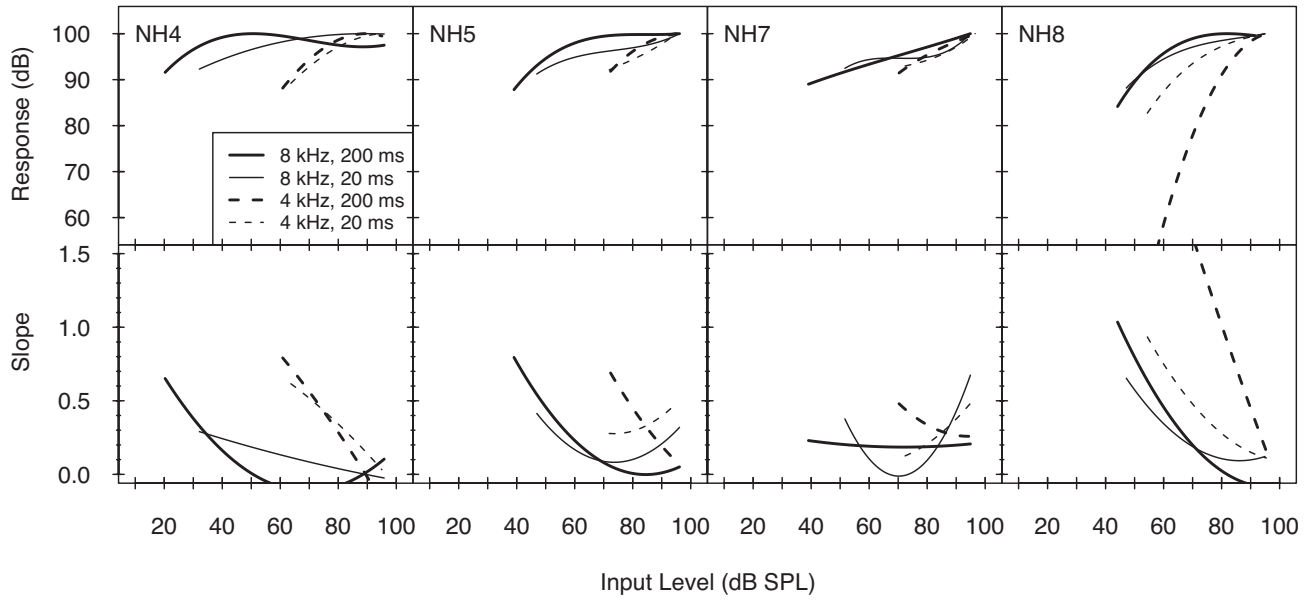

FIG. 12. Response functions (top panels) and response-function slopes (bottom panels) derived from the data of experiment 4. 
200-ms M1 and 0.14 for the 20-ms M1. Previous estimates of the mid-level compression exponent at $4000 \mathrm{~Hz}$ using the signal-vary AFM technique (with a long-duration M1) range from 0.17 (Plack and O'Hanlon, 2003) to 0.21 (Plack et al., 2006; Plack et al., 2008). The masker-signal interval conditions produced very low estimates of the compression exponent, 0.08 for the 200-ms M1 and 0.09 for the 20-ms M1 at $4000 \mathrm{~Hz}$ (experiment 2), and 0.13 for the 200-ms M1 and 0.16 for the $20-\mathrm{ms} \mathrm{M} 1$ at $8000 \mathrm{~Hz}$ (experiment 4). The lower estimates compared to the sensation-level conditions (experiment 1) could be because the use of a low-level signal limited off-frequency listening and the basalward shift of the traveling wave, both of which tend to linearize the estimated response function (Oxenham and Plack, 1997; Nelson et al., 2001).

The TMC technique also uses a fixed low-level signal, but the compression exponent estimates are greater than those reported here, being closer to 0.2 (Nelson et al., 2001; Lopez-Poveda et al., 2003; Plack and Drga, 2003; Rosengard et al., 2005; Williams and Bacon, 2005). However, if the TMC technique underestimates compression because it incorrectly assumes a linear off-frequency response, this could account for the discrepancy. The exponent estimates from experiment 2 are roughly half those from the TMC studies. If it is assumed that off-frequency compression is about $2: 1$, as suggested by the present AFM results, then the TMC exponent values are overestimates by a factor of two. On the other hand, direct measurements of BM vibration in other mammals suggest $\mathrm{CF}$ compression exponents of about 0.2 (Ruggero, 1992; Murugasu and Russell, 1995; Ruggero et al., 1997; Rhode and Recio, 2000), more similar to the TMC measures than to the present AFM measures with a low-level signal.

The present experiments were conducted in two phases. In phase 1 equally effective maskers were determined, and in phase 2 maskers in the combined condition were presented at their equally effective relative levels. However, except for experiment 3, combined masker conditions were also included in phase 1 , in which the maskers were presented at equal SPLs. Hence, it is possible to generate response function estimates from the phase 1 data alone. The advantage to using equally effective maskers in the combined-masker conditions in phase 2 is that one masker cannot dominate the masking and thereby limit the reliability of the technique. However, for some of the conditions tested in the experiments it could be argued that phase 2 was not necessary, except to increase the reliability of the response function estimate by increasing the number of data points. In particular, in experiment 2 for a 20-ms M1 and a 10-ms M2, thresholds for M1 and M2 measured individually are similar, suggesting that equal level approximates equal effectiveness in the combined conditions. These might be appropriate stimuli to use in a more efficient version of the AFM technique, without phase 2 .

\section{B. Off-frequency compression}

All four experiments provided evidence of compression in the auditory system for frequency components an octave or more below CF. Off-frequency compression exponent estimates vary between the experiments and conditions, ranging from 0.32 to 0.64 when averaged across listeners and across level. These values are broadly similar to the estimate of 0.5 (2:1 compression) reported by Lopez-Poveda and Alves-Pinto (2008) for a $4000-\mathrm{Hz}$ signal and a $2200-\mathrm{Hz}$ masker. However, in experiment 3 of the present study a compression exponent of 0.54 was found for a $4000-\mathrm{Hz}$ signal and a $1500-\mathrm{Hz}$ masker, whereas Lopez-Poveda and Alves-Pinto reported a linear response for a $1600-\mathrm{Hz}$ masker. Physiological results from other mammals are consistent in showing that the BM response in the base of the cochlea is linear for signals an octave below CF (Ruggero, 1992; Ruggero et al., 1997; Russell and Nilsen, 1997).

Hence, both on- and off-frequency compression estimates are greater when measured with the AFM technique in human listeners than would be expected based on direct measures of the BM response in other animals. A possible explanation for the additional compression observed in the present data is that there is a compressive non-linearity central to the $\mathrm{BM}$ that contributes to the effect of combining two maskers in the AFM technique. Such a non-linearity would affect both on- and off-frequency responses, assuming that masking of the signal is determined by BM excitation at the signal place. The non-linearity could result from neural saturation. However, the fact that listeners with moderate cochlear hearing loss tend to show linear additivity effects (Oxenham and Moore, 1995; Plack et al., 2008) suggests that the nonlinearity is cochlear in origin. A more plausible hypothesis is that the non-linearity resides in the transduction process in the inner hair cells (IHCs) (Lopez-Poveda et al., 2005). The growth in IHC receptor potential with level shows a compressive response for $2000-\mathrm{Hz}$ tones at the $3800-\mathrm{Hz}$ place, with a compression ratio of $2: 1$ or greater at high levels, although a more linear response is observed at lower levels (Cheatham and Dallos, 2001). This is broadly consistent with the estimates of off-frequency compression reported here, and is also broadly consistent with the finding that, for most listeners, off-frequency compression increases with increasing level. In their model of IHC function, Lopez-Poveda and Eustaquio-Martin (2006) relate this compression to basolateral potassium currents (Kros and Crawford, 1990), and confirm that IHC compression affects all frequencies equally. If dysfunction of the IHCs leads to a complete loss of IHC compression, then this might explain why hearing-impaired listeners show linear masker additivity. Loss of IHC compression could also explain the finding that the slopes of off-frequency TMCs (plots of masker threshold level against masker-signal interval) are steeper for normal than for impaired ears (Plack et al., 2004; Lopez-Poveda et al., 2005; Rosengard et al., 2005), and the finding that the effect of masker duration on absolute threshold is greater for normal ears than for impaired ears (Plack and Skeels, 2007). Hence, one explanation for these three findings is that cochlear hearing loss results from $\mathrm{OHC}$ and IHC dysfunction. OHC dysfunction reduces on-frequency compression, and IHC dysfunction reduces both on- and off-frequency compression. However, it should be emphasized that all three of these effects are also consistent with a normal IHC response, and a 
loss of on- and off-frequency compression on the BM due to $\mathrm{OHC}$ dysfunction.

In the TMC technique it is often assumed that increased compression will manifest as an increase in the steepness of the TMC. For each increase in masker-signal interval, the masker level at threshold is increased to compensate for the decay of forward masking. The increase in physical level will be greater if the masker is compressed. Hence, if offfrequency compression increases with level, as suggested by the IHC hypothesis, we might expect TMC slope to increase with increasing masker level (and hence with increasing masker-signal interval). This is not usually observed. Indeed, the slope of off-frequency TMCs is sometimes observed to decrease with increasing interval. However, the argument for increasing slope depends on an exponential post-cochlear decay of forward masking (so that, in the absence of compression, the masker level in $\mathrm{dB}$ is linearly related to maskersignal interval). If the post-cochlear decay (in $\mathrm{dB} / \mathrm{ms}$ ) becomes shallower with increasing interval, then this might explain the lack of increase in TMC slope.

Together with the results of Lopez-Poveda and AlvesPinto (2008), the present data raise concerns about the validity of the assumption of off-frequency linearity used in the TMC and GOM techniques. However, it should be noted that if the additional compression identified here results from processes in the IHC as suggested above, then the GOM and TMC techniques may still be valid measures of BM compression (since the effects of IHC compression will likely be the same for on- and off-frequency maskers, and hence the differential masking growth for on- and off-frequency masker will reveal differences in BM compression).

Wojtczak and Oxenham (2009) have raised doubts about another assumption underlying the TMC technique. They provided evidence that the decay of BM excitation for equally effective maskers is slower for an off-frequency masker at high levels (92 dB SPL) than for an equally effective on-frequency masker. The decay at lower levels $(83 \mathrm{~dB}$ SPL for the off-frequency masker) was the same on and off frequency. The TMC technique assumes that the decay of excitation at the signal place is independent of stimulation frequency. Notice that if the rate of masker decay of the off-frequency masker decreases with increasing level then this would increase the effectiveness (and hence reduce the threshold level) of maskers as masker level is increased. The level difference between conditions, and the estimate of compression in the AFM technique, would be reduced (compression exponent increased). Hence, the findings of Wojtczak and Oxenham, if confirmed, could imply that the present results are underestimates of off-frequency compression.

\section{Effects of efferents}

Two types of efferent activation were investigated in the present study: the stapedius reflex and the MOCR. Contraction of the stapedius muscle has its largest effect on frequencies below $1000 \mathrm{~Hz}$, and is thought to have no effect on frequencies above $2000 \mathrm{~Hz}$ in humans (Rosowski and Relkin, 2001). It is just possible that contraction of the stapedius muscle in response to the high masker levels could have made a small contribution to the off-frequency compression observed for the $1500-\mathrm{Hz}$ and $2000-\mathrm{Hz}$ maskers in experiments 1-3. However, off-frequency compression for an $8000-\mathrm{Hz}$ signal and $4000-\mathrm{Hz}$ masker in experiment 4 was similar to that measured in experiments $1-3$. Hence, it seems unlikely that the stapedius reflex had a large effect on the present results.

In experiment 1, decreasing M1 duration from 200 to 20 ms decreased the slope of the response functions slightly, suggesting a possible influence of the MOCR. No effect of M1 duration was observed in the other experiments. A reduction in compression is usually associated with a reduction in gain. The AFM technique does not provide a direct estimate of gain, since it only allows an estimation of the slope of the response functions. However, in the TMC technique, the maximum gain of the BM can be estimated by measuring the difference between the on- and off-frequency thresholds for low levels of the on-frequency masker (for which it is assumed that the masker is in the linear region of the BM response function). Because similar on- and off-frequency masking data were measured here, the same analysis can be performed. It was decided that the best conditions for estimating gain are the equal-level combined-masker conditions for the 0 -ms gap from experiment 2, since the on-frequency levels are generally low. However, even for these conditions the levels are sometimes above the linear region of the response function, which will tend to produce an underestimate of maximum gain. For these conditions, the average threshold differences, off minus on, for the 0-ms masker-signal interval are $46.58 \mathrm{~dB}(\mathrm{SE}=0.36)$ for the 200-ms $\mathrm{M} 1$, and $42.98 \mathrm{~dB}(\mathrm{SE}=3.10)$ for the $20-\mathrm{ms} \mathrm{M} 1$. Hence, there is no evidence for reduced gain in the long masker condition, although of course the levels were relatively low, and perhaps not sufficient to elicit a measurable MOCR.

As described in the introduction, it has been suggested that at least part of the effect of combining maskers in the AFM technique could be a consequence of linearization of the BM response by the MOCR triggered by the first masker (Krull and Strickland, 2008; Jennings et al., 2009). Under this hypothesis, the first masker triggers the MOCR, thereby reducing the compression applied to the second masker and the signal. Since the signal is usually lower in level than the masker at forward-masked threshold, the effect of the linearization is to increase the level difference between the masker and signal on the BM. Put another way, the gain applied to the signal is reduced more by the MOCR than the gain applied to the masker. It follows that, even though the effect of MOCR is to linearize the BM response, the MOCR triggered by the first masker could cause an increase in masked threshold in the combined condition, accounting for the large increase in masking observed when two forward maskers are combined.

The on-frequency results from experiment 1 do not support the extreme version of this hypothesis, that the effect of combining two maskers is entirely dependent on the MOCR, since the effect of combining two maskers was greatest with the shortest M1 duration. In other words, the effect of combining the maskers increased as the potential efferent stimulation decreased. If the increase in masking in the combined 
condition were entirely dependent on the MOCR one would expect the reverse effect. However, these data do not rule out the possibility that a combination of MOCR (mainly long M1) and additivity (mainly short M1) could account for the effects.

The mechanism proposed by Jennings et al. (2009) could also potentially explain the effects of combining the off-frequency maskers. If so, this could mean that the explanations in terms of BM or IHC compression described in Section VIII B are not required. To test this, compression estimates were made from the off-frequency masker thresholds for the shortest M2-signal delay $(0 \mathrm{~ms})$ from experiments 2 and 4. For the 20-ms M1 and for this M2-signal delay, there should have been very little MOCR activity at the time of presentation of the signal. To simplify the analysis, and reduce the number of free parameters in the model, compression was assumed to be constant:

$$
f(x)=a x^{c},
$$

where $x$ is intensity and $c$ is the compression exponent. Following the procedure described in Section III B, this equation was substituted into Eq. (10), and the single masker thresholds used to predict the combined masker threshold. As before, the phase 1 and phase 2 results were fit together with the same function to give two combined masker $(\mathrm{M} 1+\mathrm{M} 2)$ predictions per listener. $c$ was varied adaptively to find the exponent that best fit the data.

For experiment 2 (4000-Hz signal, 2000-Hz maskers), the mean off-frequency compression exponents calculated in this way are $0.61(\mathrm{SE}=0.086)$ for the $200-\mathrm{ms} \mathrm{M} 1$, and 0.51 $(\mathrm{SE}=0.12)$ for the $20-\mathrm{ms} \mathrm{M} 1$. These values do not differ significantly from each other, but both are significantly less than $1(\mathrm{p}<0.01$ in each case). For experiment $4(8000-\mathrm{Hz}$ signal, $4000-\mathrm{Hz}$ maskers), NH8 has a very high $c$ value (3.29, more than $1 \mathrm{SD}$ from the mean) for the 20-ms M1, resulting from the anomaly that the combined threshold was greater than the single masker thresholds for the equally effective conditions (see Fig. 11, bottom right panel). The mean off-frequency compression exponents are 0.51 (SE $=0.036)$ for the 200-ms M1, and $1.06(\mathrm{SE}=0.74)$ for the 20-ms M1. However, these means are not significantly different. Excluding NH8, the story is rather different, with means of $0.51(\mathrm{SE}=0.051)$ for the 200-ms M1, and 0.32 $(\mathrm{SE}=0.060)$ for the $20-\mathrm{ms} \mathrm{M} 1$.

Overall then, the short M1 data suggest that the nonlinear effect of combining two off-frequency maskers is not dependent on the MOCR. Hence, it is more likely that the data reflect off-frequency compressive processes in the auditory periphery, as discussed in Section VIII B. It is important to emphasize, however, that the present results do not imply that the MOCR elicited by a long M1 has little effect on the underlying BM response functions. For example, it is still possible that the apparent on- and off-frequency compression observed at long M1 durations could be caused by the MOCR. Rather, the results suggest that the AFM technique is relatively immune to these influences, such that whatever effect the MOCR has does not greatly affect the estimates of slope. However, it is perhaps advisable that future AFM experiments use a short M1 duration to minimize any potential confound.

\section{CONCLUSIONS}

(i) A masker-vary version of the AFM technique was introduced. Compression at different levels was estimated using different overall signal levels, or different masker-signal intervals. Results are consistent with those produced by the original signal-vary AFM technique, showing considerable on-frequency compression over a range of signal and masker levels. Compression estimates increased when a fixed low-level signal and a range of masker-signal intervals were used.

(ii) Approximately 2:1 off-frequency compression was observed for a $2000-\mathrm{Hz}$ or $1500-\mathrm{Hz}$ masker at the $4000-\mathrm{Hz}$ place. 2:1 compression was also observed for a $4000-\mathrm{Hz}$ masker at the $8000-\mathrm{Hz}$ place, suggesting that the off-frequency compression was not a result of the stapedius reflex.

(iii) In some cases, on-frequency compression increased slightly as masker duration was decreased, suggesting that the MOCR triggered by a long first masker may affect some compression estimates using the AFM technique.

\section{ACKNOWLEDGMENTS}

The research was supported by BBSRC (U.K.) Grant No. BB/D012953/1. We are very grateful to the Associate Editor (Magdalena Wojtczak), Enrique Lopez-Poveda, and an anonymous reviewer for their insightful and constructive comments on an earlier version of the manuscript.

${ }^{1}$ Throughout this article, the term "technique" is used to refer to the complete procedure of collecting data and deriving response functions from the data.

Backus, B. C., and Guinan, J. J. (2006). "Time course of the human medial olivocochlear reflex," J. Acoust. Soc. Am. 119, 2889-2904.

Cheatham, M. A., and Dallos, P. (2001). "Inner hair cell response patterns: Implications for low-frequency hearing," J. Acoust. Soc. Am. 110, 20342044.

Cooper, N. P., and Guinan, J. J. (2006). "Efferent-mediated control of basilar membrane motion," J. Physiol. (London) 576, 49-54.

Hicks, M. L., and Bacon, S. P. (1999). "Psychophysical measures of auditory nonlinearities as a function of frequency in individuals with normal hearing," J. Acoust. Soc. Am. 105, 326-338.

James, A. L., Harrison, R. V., Pienkowski, M., Dajani, H. R., and Mount, R. J. (2005). "Dynamics of real time DPOAE contralateral suppression in chinchillas and humans," Int. J. Audiol. 44, 118-129.

Jennings, S. G., Strickland, E. A., and Heinz, M. G. (2009). "Precursor effects on behavioral estimates of frequency selectivity and gain in forward masking," J. Acoust. Soc. Am. 125, 2172-2181.

Johnson-Davies, D. B., and Patterson, R. D. (1979). "Psychophysical tuning curves: Restricting the listening band to the signal region," J. Acoust. Soc. Am. 65, 765-770.

Kros, C. J., and Crawford, A. C. (1990). "Potassium currents in inner hair cells isolated from the guinea-pig cochlea," J. Physiol. 421, 263-291.

Krull, V., and Strickland, E. (2008). "The effect of a precursor on growth of forward masking," J. Acoust. Soc. Am. 123, 4352-4357.

Levitt, H. (1971). "Transformed up-down methods in psychoacoustics," J. Acoust. Soc. Am. 49, 467-477.

Liberman, M. C., and Brown, M. C. (1986). "Physiology and anatomy of 
single olivocochlear neurons in the cat," Hear. Res. 24, 17-36.

Liberman, M. C., Puria, S., and Guinan, J. J. (1996). "The ipsilaterally evoked olivocochlear reflex causes rapid adaptation of the $2 f_{1}-f_{2}$ distortion product otoacoustic emission," J. Acoust. Soc. Am. 99, 3572-3584.

Lopez-Poveda, E. A., and Alves-Pinto, A. (2008). "A variant temporalmasking-curve method for inferring peripheral auditory compression," J. Acoust. Soc. Am. 123, 1544-1554.

Lopez-Poveda, E. A., and Eustaquio-Martin, A. (2006). "A biophysical model of the inner hair cell: The contribution of potassium currents to peripheral auditory compression,” J. Assoc. Res. Otolaryngol. 7, 218-235.

Lopez-Poveda, E. A., Plack, C. J., and Meddis, R. (2003). "Cochlear nonlinearity between 500 and $8000 \mathrm{~Hz}$ in listeners with normal hearing," J. Acoust. Soc. Am. 113, 951-960.

Lopez-Poveda, E. A., Plack, C. J., Meddis, R., and Blanco, J. L. (2005). "Cochlear compression between 500 and $8000 \mathrm{~Hz}$ in listeners with moderate sensorineural hearing loss," Hear. Res. 205, 172-183.

Moore, B. C. J., Vickers, D. A., Plack, C. J., and Oxenham, A. J. (1999). "Inter-relationship between different psychoacoustic measures assumed to be related to the cochlear active mechanism," J. Acoust. Soc. Am. 106, 2761-2778.

Murugasu, E., and Russell, I. J. (1995). "Salicylate ototoxicity: The effects on basilar membrane displacement, cochlear microphonics, and neural responses in the basal turn of the guinea pig cochlea," Aud. Neurosci. 1, 139-150.

Nelson, D. A., and Schroder, A. C. (2004). "Peripheral compression as a function of stimulus level and frequency region in normal-hearing listeners," J. Acoust. Soc. Am. 115, 2221-2233.

Nelson, D. A., Schroder, A. C., and Wojtczak, M. (2001). “A new procedure for measuring peripheral compression in normal-hearing and hearingimpaired listeners," J. Acoust. Soc. Am. 110, 2045-2064.

Oxenham, A. J. (1997). "Increment and decrement detection in sinusoids as a measure of temporal resolution," J. Acoust. Soc. Am. 102, 1779-1790.

Oxenham, A. J., and Moore, B. C. J. (1995). "Additivity of masking in normally hearing and hearing-impaired subjects," J. Acoust. Soc. Am. 98, 1921-1934.

Oxenham, A. J., and Plack, C. J. (1997). "A behavioral measure of basilarmembrane nonlinearity in listeners with normal and impaired hearing," J. Acoust. Soc. Am. 101, 3666-3675.

Plack, C. J., Carcagno, S., and Oxenham, A. J. (2007). "A further test of the linearity of temporal summation in forward masking," J. Acoust. Soc. Am. 122, 1880-1883.

Plack, C. J., and Drga, V. (2003). "Psychophysical evidence for auditory compression at low characteristic frequencies," J. Acoust. Soc. Am. 113, $1574-1586$

Plack, C. J., Drga, V., and Lopez-Poveda, E. A. (2004). "Inferred basilarmembrane response functions for listeners with mild to moderate sensorineural hearing loss," J. Acoust. Soc. Am. 115, 1684-1695.

Plack, C. J., and O'Hanlon, C. G. (2003). "Forward masking additivity and auditory compression at low and high frequencies," J. Assoc. Res. Otolaryngol. 4, 405-415.

Plack, C. J., Oxenham, A. J., and Drga, V. (2006). "Masking by inaudible sounds and the linearity of temporal summation," J. Neurosci. 26, 87678773 .

Plack, C. J., Oxenham, A. J., Simonson, A., O’Hanlon, C. G., Drga, V., and Arifianto, D. (2008). "Estimates of compression at low and high frequencies using masking additivity in normal and impaired ears," J. Acoust. Soc. Am. 123, 4321-4330.

Plack, C. J., and Skeels, V. (2007). "Temporal integration and compression near absolute threshold in normal and impaired ears," J. Acoust. Soc. Am. 122, 2236-2244.

Rabinowitz, W. M. (1977). Acoustic-Reflex Effects on the Input Admittance and Transfer Characteristics of the Human Middle Ear (Massachusetts Institute of Technology, Boston).

Rhode, W. S. (1971). "Observations of the vibration of the basilar membrane in squirrel monkeys using the Mössbauer technique," J. Acoust. Soc. Am. 49, 1218-1231.

Rhode, W. S., and Cooper, N. P. (1996). "Nonlinear mechanics in the apical turn of the chinchilla cochlea in vivo," Aud. Neurosci. 3, 101-121.

Rhode, W. S., and Recio, A. (2000). "Study of mechanical motions in the basal region of the chinchilla cochlea," J. Acoust. Soc. Am. 107, 33173332.

Robles, L., Ruggero, M. A., and Rich, N. C. (1986). "Basilar membrane mechanics at the base of the chinchilla cochlea. I. Input-output functions, tuning curves, and phase responses," J. Acoust. Soc. Am. 80, 1364-1374.

Rosengard, P. S., Oxenham, A. J., and Braida, L. D. (2005). "Comparing different estimates of cochlear compression in listeners with normal and impaired hearing," J. Acoust. Soc. Am. 117, 3028-3041.

Rosowski, J. J., and Relkin, E. M. (2001). "Introduction to the analysis of middle ear function," in Physiology of the Ear, edited by A. F. Jahn and J. Santos-Sacchi (Singular, San Diego), pp. 161-190.

Ruggero, M. A. (1992). "Responses to sound of the basilar membrane of the mammalian cochlea," Curr. Opin. Neurobiol. 2, 449-456.

Ruggero, M. A., and Rich, N. C. (1991). "Furosemide alters organ of Corti mechanics: Evidence for feedback of outer hair cells upon the basilar membrane," J. Neurosci. 11, 1057-1067.

Ruggero, M. A., Rich, N. C., Recio, A., Narayan, S. S., and Robles, L. (1997). "Basilar-membrane responses to tones at the base of the chinchilla cochlea," J. Acoust. Soc. Am. 101, 2151-2163.

Russell, I. J., and Nilsen, K. E. (1997). "The location of the cochlear amplifier: Spatial representation of a single tone on the guinea pig basilar membrane," Proc. Natl. Acad. Sci. U.S.A. 94, 2660-2664.

Warr, W. B., and Guinan, J. J. (1979). "Efferent innervation of the organ of corti: Two separate systems," Brain Res. 173, 152-155.

Williams, E. J., and Bacon, S. P. (2005). "Compression estimates using behavioral and otoacoustic emission measures," Hear. Res. 201, 44-54.

Wojtczak, M., and Oxenham, A. J. (2009). "Pitfalls in behavioral estimates of basilar-membrane compression in humans," J. Acoust. Soc. Am. 125, 270-281.

Yasin, I., and Plack, C. J. (2003). "The effects of a high-frequency suppressor on tuning curves and derived basilar membrane response functions," J. Acoust. Soc. Am. 114, 322-332.

Yates, G. K. (1995). "Cochlear structure and function," in Hearing, edited by B. C. J. Moore (Academic, San Diego), pp. 41-74. 